\title{
Factors associated with coverage of praziquantel for schistosomiasis control in the community- direct intervention (CDI) approach in Mali (West Africa)
}

Abdoulaye Dabo ${ }^{1 *}$, Boubacar Bary ${ }^{1}$, Bourema Kouriba ${ }^{1}$, Oumar Sankaré ${ }^{2}$ and Ogobara Doumbo

\begin{abstract}
Background: Despite the progress made in the control of Neglected Tropical Diseases (NTD), schistosome and soil-transmitted helminth infections are far from being effectively managed in many parts of the world. Chemotherapy, the key element of all control strategies, is faced with some difficulties in terms of access to treatment. Our study aims to describe the factors involved in the success or failure of the community-directed intervention (CDI) approach through control programmes, which aims to achieve consistent high coverage at affordable and sustainable costs in endemic areas.
\end{abstract}

Methods: The CDI approach was carried out from December 2007 to October 2008 in ten villages of the district of Diéma, Mali. At inclusion, each child part of the study's sample was interviewed and submitted for a physical examination. The study focused on: data collection, treatment of the eligible population, evaluation of the treatment coverage, performance of community drug distributors (CDDs), and the involvement and perception of populations.

Results: A total of 8,022 eligible people were studied with a mean coverage rate of $76.7 \%$. Using multiple regression, it was determined that receiving praziquantel as treatment was associated with five factors: belonging to the Fulani or Moorish ethnic minority versus the Bambara/Soninke, use of the central versus the house-to-house drug distribution mode, the ratio of the population to the number of CDDs, the lack of supervision and belonging to the age group of 15 years or above $(p<0.05)$. As well as that, it was found that the presence of parallel community-based programmes (HIV, tuberculosis) that provide financial incentives for community members discouraged many CDDs (who in most cases are volunteers) to participate in the CDI approach due to a lack of incentives.

Conclusions: The findings indicate that the success of the CDI approach depends on, amongst other things, the personal characteristics of the respondents, as well as on community factors.

Keywords: Schistosomiasis, Praziquantel, Community-directed intervention, Coverage rate, Diéma, Mali

\footnotetext{
* Correspondence: adabo@icermali.org

'Department of Epidemiology of Infectious Diseases, Faculty of Medicine, Pharmacy and Dentistry, UMI 3189, University of Sciences, Techniques and Technologies of Bamako, Box 1805, Bamako, Mali

Full list of author information is available at the end of the article
} 


\section{Multilingual abstracts}

Please see Additional file 1 for translations of the abstract into the six official working languages of the United Nations.

\section{Background}

Schistosomiasis remains to be a major public health problem in many parts of Sub-Saharan Africa. It is estimated that, globally, there are 207 million people infected with one of the three major species of schistosomes [1], more than $90 \%$ of which occur in Sub-Saharan Africa [1,2]. A review of the relationship between infection and clinical morbidity in Sub-Saharan Africa estimates that deaths due to schistosomiasis may be as high as 200,000 per year [3]. In Mali, schistosomiasis is mainly associated with water resource development around dam construction. The prevalence rate of the disease varies from $40 \%$ in savannah villages to $80-90 \%$ in Office du Niger and Bandiagara [4-6].

For the past 20 years, strategies have been developed to control schistosomiasis in endemic areas. The key elements of these strategies were to control morbidity especially by selective treatment of heavy infections through regular treatment of high-risk groups (schoolchildren, fishermen and irrigation workers). It has been shown that mass treatment and health education of the population have significantly reduced transmission and morbidity in countries and regions such as Brazil, Venezuela, China, Indonesia, the Philippines, the Maghreb and the Middle East [7]. Despite this progress, schistosomiasis is far from being controlled in many parts of world. Because of the difficulties that exist in relation to access to treatment, as well as the non-involvement of communities in the process of the distribution and treatment of schistosomiasis, these strategies have failed to reach their objectives. Today, less than $10 \%$ of the treatment-eligible populations living in endemic regions of Africa, Asia and the Americas receive annual treatment for schistosomiasis, intestinal helminth infections and/or trachoma [2]. To improve access to treatment at affordable and sustainable costs and, where possible, to improve existing programmes, community-directed intervention (CDI) has been presented as an alternative strategy to control schistosomiasis and soil-transmitted helminths (STHs). This approach has been adopted in several settings [8-10] and has been successful in many countries as part of the African Programme for Onchocerciasis Control (APOC) [11,12], as well as for lymphatic filariasis control [13,14]. In Mali, despite the Schistosomiasis and Soil-transmitted Helminths National Control Programme (PNSHs) adopting the CDI approach, the programme has faced some difficulties which led to very low coverage $(<60 \%)$ in some districts (oral communication of the PNLSH's coordinator).
The overall objective of this study was to identify the prominent factors affecting the programme's failure to improve access to treatment for all those at risk of both overt and subtle morbidity due to schistosomiasis in the health district of Diéma, Mali.

\section{Methods \\ Experimental design and methods \\ Overall study design}

The study focused on $i$ ) census and description of social and demographical data at baseline, ii) choice and selection of CDDs, iii) the social and cultural context of the CDI approach, and iv) evaluation of the study outcomes (treatment coverage, performance of CDDs, and involvement and perception of populations) one month after drug distribution started, and the issues related to side effects 24 hours after drug administration. The study was conducted between December 2007 and November 2008.

\section{Sampling design and sample size}

The study's design was an exploratory and descriptive trial at the community level. The study unit, determined by the implementation level of the health services, was a village. The study population was comprised of an entire village population. To measure the success of this design, four villages were selected based upon the prevalence of schistosomiasis, the functionality of the health services and village accessibility. First, all functioning health areas in the 13 villages were listed. Ten villages were then selected from these health centres based on the accessibility and the acceptance of the population to participate in the study. The sample size of the eligible population (children five to 15 years of age who would provide urine and stool samples for analysis) was calculated according to the total population in each village, the precision (5\%), the attended prevalence in the study site (60\%) and the risk (alpha 5\%) as described [15]. According to the statistical and demographical projection data, the population size in all ten villages was 9,806 . However, to get more information on the population and the number of tablets required, the research team and the community drug distributors (CDDs) determined the study population after a new census was completed and the population of the villages was confirmed to be 8,022 .

\section{Study sites and health systems}

In Mali, schistosomiasis is prevalent all over the country, but is endemic only in four (35 districts) of the eight regions. Following the Schistosomiasis Control Initiative (SCI), the CDI strategy was successfully implemented in the entire endemic district for schistosomiasis in Mali since 2005. However, the CDI strategy failed in the 
district of Diéma, in the north-west of Mali, where the coverage rate was lower than 60\% [16]. Diéma was selected as a study site because of the CDI strategy's failure to raise coverage, as expected, in this district compared to the other districts.

The study took place in ten endemic villages in Diéma (Figure 1). All the villages are accessible by car and range in distance from seven to 20 kilometers from Diéma.

Health services in Diéma are organised in two levels: the community and the district level. Services at the community level are delivered by a primary health care centre $(\mathrm{CHC})$. They are managed by selected community representatives and led by a health officer appointed by the government. The management of the district health services (DHS) is done by the district management team and headed by the District Medical Officer (DMO). The $\mathrm{CHC}$ management team defers to the DMO as the overall leader of the district health system. The access to health facilities varied from $\$ 0.4$ to $\$ 1$ per visit. In some villages, the health charges were sometimes evaluated into millet, rice, peanuts and/or poultry. Praziquantel (PZQ) is not always available at the health centre - it is only available during the mass drug distribution campaigns. Normally, patients suffering from schistosomiasis were examined (using questionnaires at the community level, or using a microscope for stool and urine examination at the district reference health centre). After routine examination, each patient would pay for the PZQ when he/she tested positive.

The social organisation of these communities was primarily hierarchical, based on lineage. The leadership includes a village head and the village council comprising of the heads of the village families. The village head and the council are responsible for the general administration and decision making of the village. At the community level, socio-economic activities are done according to sex and age groups. In some villages, because of the weight of tradition, women and men cannot mingle or attend meetings together. The illiteracy rate is generally high $(70 \%)$ even if the policy of adult education in local languages is well developed. The main ethnic groups are Soninke and Bambara, and there are minority groups such as Fulani and Moorish. Islam is the major religion. The most common occupation is farming and cattle rearing, with close proximity to standing water sources. Young men in the region migrate extensively within, and outside, the country for economic purposes.

There are two major seasons: a rainy season from June/July to September/October, and a dry season which

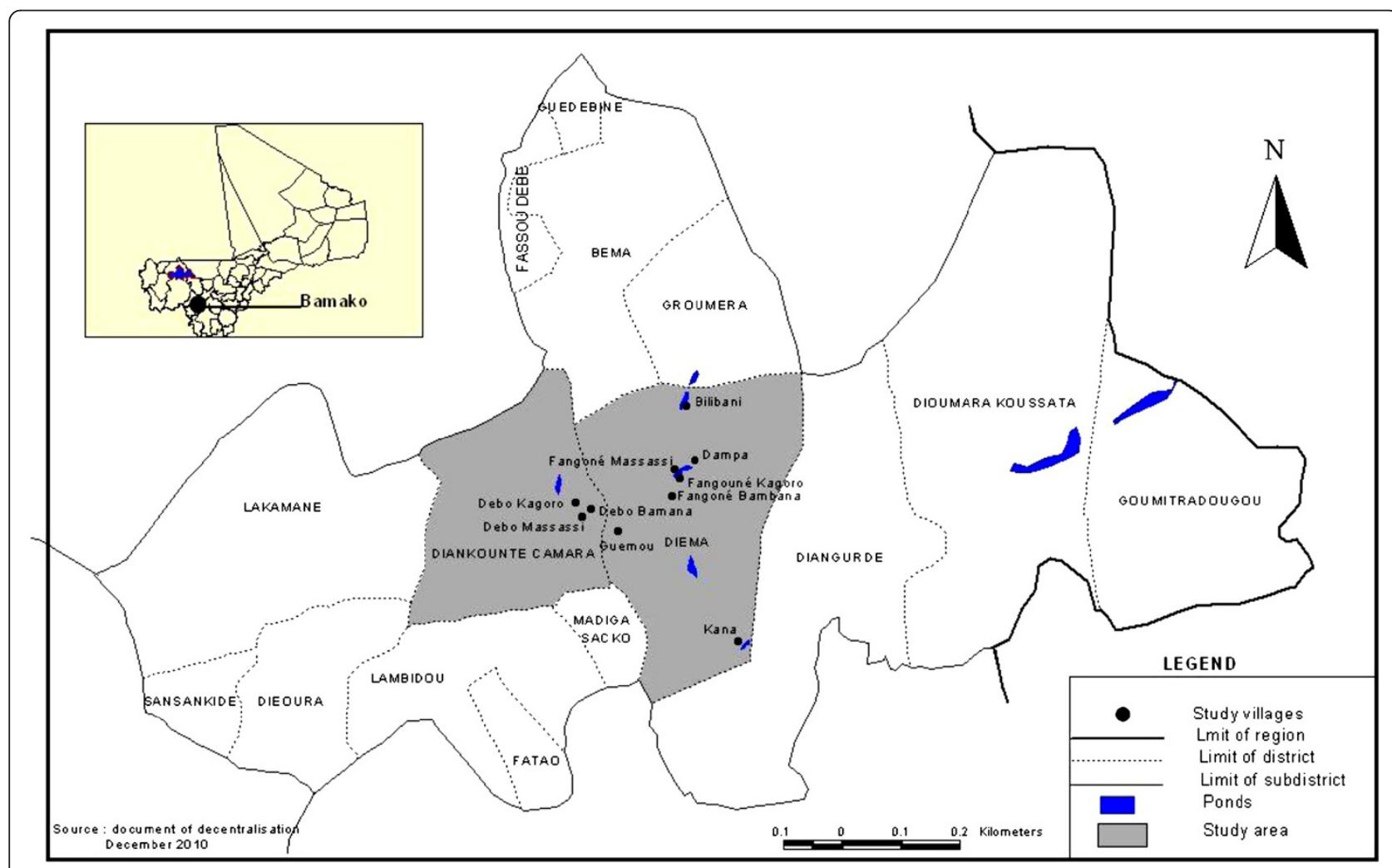

Figure 1 Localization of the ten study villages $(*)$ in the district of Diéma, Mali. 
is divided into a cold season from November to January and a hot season from February to May. Schistosomiasis is seasonally transmitted from July to December.

\section{Techniques of the study Choice and selection of community-directed health workers (CDHWs)}

Community drug distributors (CDDs) were proposed for, and selected by, the population. One CDD per 125 inhabitants was selected and trained. Prior to the training of the CDDs, each team conducted health education using posters and/or verbal presentations in every CDI study village to address the following issues: knowledge of the disease, knowledge of treatment, attitude to treatment, attitude to disease, attitude to the distributor, attitude to good keeping and information/record sharing. Following the training, the CDDs were expected to be able to describe the major clinical manifestations of schistosomiasis, the different methods of treatment (house-to-house versus central point), and to have a working knowledge of the methods of carrying out the following PZQ distribution-related activities: community mobilisation, household enumeration, guidelines on the period and the duration of treatment, guidelines for the exclusion criteria, correct dosage using height, recognition of minor reactions and when to refer adverse reactions, basic record keeping, and to identify and report the issues encountered during the distribution. These guidelines could be modified when necessary. The dosage of PZQ for eligible members of the population was determined using a person's height measurement [17]. An easy-to-transport stick was calibrated to serve as the measuring ruler. Tablets were swallowed in the presence of the distributor.

Attitude and perception of community-directed health workers (CDHWs) in the CDI strategy were scored on a scale ranging from strongly agree to uncertain to strongly disagree. Two major opinion items were considered to assess the health workers' role in CDTI: $i$ ) the CDHWs would like to participate in the training of community members to take responsibility for PZQ and albendazole (ALB) distribution, and ii) the CDHWs would like to take part in all decision-making processes concerning the distribution of PZQ and ALB at the community level.

\section{The social and cultural context of community-directed intervention (CDI)}

The CDI strategy is an approach whereby community members collectively: (i) discuss a health or developmental challenge; (ii) design the approach to implement the interventions in the community; (iii) identify the resources to accomplish the task; and (iv) plan how, when, where and by whom it will be implemented. Like in the control of onchocerciasis [18], one aspect of the CDI strategy for schistosomiasis control through PZQ distribution is the appreciation and use of socio-cultural aspects of the communities, such as the social structures, legal system, resource mobilisation and sharing systems. Selection of many CDDs and CDHWs is vital for achieving high-drug coverage and integrating health programmes within the CDI strategy, respectively. Having, at least, both female and male CDHWs at the kinship level ensured prompt, equitable and quality healthcare delivery for all categories of community members. So, in some villages, females were not eligible to be CDDs due to the social and cultural context of that village.

In terms of why there was low morale amongst health workers and CDDs, most claimed to have been busy at the health units or at home, respectively, but many also had low morale because of their poor remuneration. However, it was not clear if CDHWs and CDDs, when involved in other health and development activities, could continue distributing PZQ effectively and efficiently, whether increased responsibilities would result in a higher drop-out rate and whether they would demand monetary incentives as a condition for services provided.

\section{Community mobilisation}

During the first visit to a village, the research team met the village head or his representative to explain the purpose of the study. The research team met the entire community at a mutually convenient date. These meetings provided an opportunity to review the problem and transmission of schistosomiasis, and the benefits of controlling the morbidity. The community engaged in a discussion regarding their experience with the disease, snails, haematuria and systemic symptoms, such as abdominal pain. The conditions for receiving PZQ and ALB were outlined, procedures were reviewed for recognising exclusion criteria (less than four years of age and severely ill), and it was discussed that only eligible persons would be treated, that the individual doses would be determined by height and that possible side effects would be recognised.

\section{Demographical assessment}

A census of the whole population in each village was performed at the onset of the study. The name of the village; the date of the census; the household identification number; the name of the head of the family; the name of the first wife followed by her children in order of decreasing age; and the sex, ethnicity, age and occupation of the other family members were recorded.

\section{Drug distribution}

The CDDs identified those eligible for treatment at the regular scheduled time of PZQ distribution. Children 
who were less than four years of age or below $94 \mathrm{~cm}$ (observation or questioning) and seriously ill individuals were deemed ineligible. The CDDs selected by villagers went from house to house (house-to-house distribution mode) or chose a central place in the village (central distribution mode) for the drug distribution. People who could not be present were examined at the house of the distributor and administered the drug there. The two drugs, PZQ and ALB, are anthelminthic drugs used by the National Control Programme, and are safe and tolerable. The dose of PZQ was determined according to the height of the patient [16] and it was suggested that patients eat before taking it. The dose of ALB was one tablet for all those eligible whatever the age.

\section{Treatment coverage determination}

The target population was the eligible population of each village. All the treated and non-treated people were recorded by the CDDs in a register. The treatment coverage rate was calculated by dividing the number of people who received PZQ and ALB by the total population of the village. The geographical coverage was determined by ethnicity, geographical position of families in the village and the individual's occupation.

\section{Evaluation of the performance of the CDDs}

The performance of the CDDs was assessed by randomly selecting interviewees from $10 \%$ of all households. All members of the sampled households were interviewed to determine if they received and were administered the drugs. In the event that no household members required therapy, explanations were recorded. The height of each individual was re-measured in order to crosscheck with the dose of the PZQ they received. People above 15 years of age were allowed to self-respond, however, for younger children, a guardian responded. The head of the household was also interviewed with respect to awareness of the drug delivery programme and its purpose.

\section{Evaluation of the community involvement and acceptability}

A questionnaire was administrated to the heads, as well as to the councillors, leaders and healers, of each village. The questionnaire determined the success of each village in implementing the activities of the CDI: how they received the research team, how they mobilised their populations for the implementation of the CDI strategy, how they selected their CDDs (number and quality), and what resources they committed to support the activities of the CDI.

\section{Record keeping}

Record keeping was standardised for all study villages. Data were collected in registry, except for interviews for which a guide was used. Each distributor had a list of all members of the villages. The following information was asked in the questionnaire: region, village, house number, name, sex, age, relationship to the head of the household, height, number of the eligible population, number of people treated, severe adverse reactions, number of tablets received, number of tablets distributed and remarks.

\section{The issues of adverse side effects}

A side effect or incidence of a symptom is defined as a symptom absent before the treatment and experienced after the treatment. Amelioration of a symptom is defined as a symptom that was experienced before the treatment and was no longer present 24 hours after the treatment. To describe the side effects associated with PZQ administration, a questionnaire was administered immediately, and 24 hours, after treatment whereby children were asked whether they needed any medical assistance or felt any of the following symptoms: dizziness, headache, sleepiness, fatigue, vertigo, abdominal pain, cramps, nausea, vomiting, diarrhoea, bloody stools, lower back pain and urticaria/rash. Meanwhile, parents or teachers were also invited to respond on behalf of their children about whether they experienced any of the stated symptoms prior to the treatment and at 24 hours after the treatment. The CDDs were trained to recognise and manage any adverse events related to PZQ and ALB administration. In the case of serious adverse events, the CDDs were invited to refer the patients to the $\mathrm{CHC}$.

\section{Data processing and analysis}

Each of the village CDDs entered all data on the population and the drugs administered. Quantitative data, including variables of acceptability, awareness of drug distribution and its purpose (by questionnaire administered to a sample of a household or their representative), the treatment coverage (characteristics of the respondents such as age, gender and ethnicity; design; number of distributors; method of CDDs' selection; CDDs' education and occupation; sex of the distributor; mode of distribution; and supervision) were entered into a computer and analysed using EPINFO 6.0 for trends and frequencies. A chi2 test was used for categorical variables, and logistic regression was used to test associations between factors and the variables they influence. $\mathrm{P}$ values less than 0.05 were considered to be significant.

Qualitative data from the process (sensitisation and planning of mass drug distribution, training and selection of CDDs, development of communication for social change (CFSC), drug procurement and distribution, and record keeping) were collected using observations notes, focus group discussions (FGDs) and in-depth interviews. They were transcribed and entered into a computer with 
TEXTBASE ALPHA. Segments were coded for thematic analysis of the process described above. The qualitative study in the form of descriptions, opinions or discussions was used to describe the beliefs and motivations that underline the access to treatment for all those at risk of schistosomiasis. The rationale of the qualitative study was to identify factors associated with the coverage rate (high and/or low rate) in order to improve the strategy. It also helped to explain or clarify findings from the analysis of the quantitative data. The qualitative study was carried out one month after the start of the drug distribution. It included a FGD and in-depth interviews.

A FGD lasting from one to two hours was led by a trained moderator. The FGD was held in five randomlyselected villages from the list of the study-site villages. In each village, ten households were randomly selected: one woman and one man (adults over 15 years) from each household (i.e., 20 respondents per village) were selected. Two groups for discussion (one for women and the other for men) were constituted. Each group was interviewed using a questionnaire aiming to capture respondents' opinions, beliefs and motives.

The in-depth interviews were structured to establish attitudes, beliefs and knowledge, and were conducted in the five selected villages. Three series of interviews were held in each village: $i$ ) the village heads and leaders, $i i$ ) the distributor(s) or the person responsible for the PZQ distribution, and iii) the members (adults) of the ten households in the villages.

All data were presented in the form of narratives, frequency tables and histograms. Frequencies, means and standard deviations were calculated and included sociodemographic variables (gender, age, household size, etc.), socio-economic statuses, as well as others.

\section{Ethical considerations}

The proposal was reviewed and approved by the Institutional Review Board (IRB) of the Faculty of Medicine, Pharmacy and Dentistry at the University of Bamako. Community consent was obtained before starting the study. A consent form (or assent form for minors) was signed by each study participant.

\section{Results}

\section{Treatment coverage of praziquantel and albendazole}

A total of 8,022 people were studied in the survey, according to the community drug distributor (CDD) registry. At the end of treatment, the CDDs documented all eligible villagers who received medication. Based on the results recorded in Table 1, the mean coverage rate was $76.7 \%(6156 / 8,022)$ (range: $64.0 \%$ to $90.7 \%)$. Nearly $80 \%(78.2 \%)$ of school-age children were reached. Overall, $60 \%$ of the study villages had a coverage rate above $75 \%$, with treatment coverage significantly differing
Table 1 Treatment coverage per village (\% in parenthesis) in the Diéma district, October 2008

\begin{tabular}{llll}
\hline Treatment villages & Total & No. treated & No. not treated \\
\hline Fangoune Kagoro & 754 & $588(78.0)$ & $190(24.3)$ \\
Fangoune Massassi & 722 & $591(81.9)$ & $131(18.1)$ \\
Fangoune Bambana & 602 & $386(64.1)$ & $216(35.9)$ \\
Dampa & 810 & $665(82.1)$ & $145(17.9)$ \\
Bilibani & 487 & $351(72.1)$ & $136(27.9)$ \\
Debo Massassi & 884 & $714(80.8)$ & $170(19.2)$ \\
Debo Bambana & 1221 & $837(68.6)$ & $384(31.4)$ \\
Debo Kagoro & 478 & $388(81.2)$ & $90(18.8)$ \\
Guemou & 1005 & $675(67.2)$ & $330(32.8)$ \\
Kana & 1059 & $961(90.7)$ & $98(9.3)$ \\
Total & 8022 & $6156(76.7)$ & $1866(23.3)$ \\
\hline
\end{tabular}

between villages $(\mathrm{p}<0.01)$. The mean age of people who received praziquantel (PZQ) and albendazole (ALB) (24.5 years) did not significantly differ from those who did not require therapy (24.7 years). There were more females $(52.2 \%)$ than males $(47.8 \%)$, especially in the age group 15-34 years $(\mathrm{p}<0.01)$. However, the coverage did not vary between the sex $(76.4 \%$ versus $76.3 \%$ for females and males, respectively). The pattern of treatment coverage did not vary significantly between sex among the different age groups $(p>0.05)$ either, except for the $35-44$ year old age group $(\mathrm{p}<0.01)$.

Analysis of the treatment coverage (those who took PZQ and ALB) by sex, age, ethnicity, mode of distribution and treatment ratio (population ration to CDDs) in eligible villagers (Table 2) showed that $78.1 \%$ of people under 15 years of age required medication compared with $75.3 \%$ of those above 15 years of age $(\mathrm{p}<0.01)$. There was a significant difference in compliance between ethnic groups: $77.3 \%$ of the 6,978 participants reported as belonging to the Bambara/Sarakolle group compared with $70.4 \%$ to the Fulani/Moorish group $(\mathrm{p}<0.01)$. In one village where the mode of distribution was centralised, $67.1 \%$ of the 1,005 eligible inhabitants received medication, compared to $78.3 \%$ of the 7,017 from villages with a house-to-house mode of distribution $(p<0.01)$. The number of distributors per community ranged from one to seven. The ratio of the village population to CDDs was commonly associated with coverage $(\mathrm{OR}=3.40$; $\mathrm{IC}=2.73-4.24)$. In some villages, other available community members helped during the distribution stage. In nine villages, where $74.2 \%$ of 7,005 people received drugs, more than 250 people were treated per CDD. This is in contrast to two villages where only 150 people were treated per CDD; $90.7 \%$ of 1,059 received the drugs. It was also found that the presence of health workers for supervision at the time of distribution led to high coverage rates $(\mathrm{p}<0.01 ; \mathrm{OR}=2.21 \mathrm{IC}: 1.92-2.55)$. 
Table 2 Treatment coverage by sex, age, ethnicity, mode of distribution and treatment ratio (population to CDDs ratio) in eligible villages in the Diéma district, October 2008

\begin{tabular}{|c|c|c|c|c|c|}
\hline Factor & No & Percent treated & $p$ & OR & $95 \% \mathrm{Cl}$ \\
\hline \multicolumn{6}{|l|}{ Sex } \\
\hline Male & 2984 & 76.4 & & & \\
\hline Female & 4168 & 76.3 & 0,97 & 1.00 & $0.90-1.11$ \\
\hline \multicolumn{6}{|l|}{ Age group } \\
\hline$<15$ years & 3026 & 78.1 & & & \\
\hline 15 and above & 4996 & 75.4 & $<0.01$ & 1.17 & $1.05-1.30$ \\
\hline \multicolumn{6}{|l|}{ Ethnicity } \\
\hline Bambara/Sarakolle & 6978 & 77.3 & & & \\
\hline Fulani/Moorish & 1044 & 70.4 & $<0.01$ & 1.43 & $1.24-1.65$ \\
\hline \multicolumn{6}{|c|}{ Mode of distribution } \\
\hline Central & 1005 & 67.2 & & & \\
\hline House-to-house & 7017 & 77.7 & $<0.01$ & 1.16 & $1.05-1.27$ \\
\hline \multicolumn{6}{|c|}{ Ratio of population to CDDs } \\
\hline 150/CDD & 1059 & 90.7 & & & \\
\hline$>=150 / C D D$ & 6963 & 74.2 & $<0.01$ & 3.40 & $2.73-4.24$ \\
\hline \multicolumn{6}{|c|}{ Presence of health workers } \\
\hline Health worker & 1950 & 85.4 & & & \\
\hline No health worker & 6072 & 73.4 & $<0.01$ & 2.21 & $1.92-2.55$ \\
\hline
\end{tabular}

Multivariate analysis using logistic regression shows that a person's ethnic group and age group, the mode of distribution, the population to CDD ratio and the presence of a health worker to supervise were all significantly associated with coverage rate increase (Table 3). Ethnic group and age group are personal characteristics of the respondents, while the mode of distribution, the population to CDD ratio and the presence of health workers at the time of distribution are community factors.

Overall, $23.3 \%(1,866 / 8,022)$ of the respondents did not receive medication at the time of treatment. From this group, 9\% were excluded because they were underage. Another $11 \%$ were eligible, but were either sick, pregnant or unaware of the distribution $(205 / 1,866)$.

Table 3 Factors associated with having received praziquantel and albendazole in the Diéma district, October 2008

\begin{tabular}{llll}
\hline Variables & OR & $\boldsymbol{p}$ & IC 95\% \\
\hline Sex & 0.97 & 0.67 & $0.87-1.08$ \\
Ethnic group & 1.34 & $<0.01$ & $1.16-1.55$ \\
Age group & 1.13 & 0.03 & $1.01-1.26$ \\
Mode of distribution & 1.45 & $<0.01$ & $1.25-1.68$ \\
Population to DCM ratio & 2.27 & $<0.01$ & $1.74-2.97$ \\
Presence of a health worker & 1.38 & $<0.01$ & $1.16-1.66$ \\
\hline
\end{tabular}

Only $1.7 \%$ refused to be treated. The pattern of absenteeism by age group showed that the problem was more common in those aged 15-24 years (Figure 2). Ethnic differences, however, did exist: only 2\% of 297 Fulani and Moorish and $10 \%$ of 1,569 Bambara and Sarakolle complained that they had not been informed. Being absent at the time of distribution was more common among the Fulani and Moorish (70.3\%) than the Bambara and Sarakolle (30.2\%).

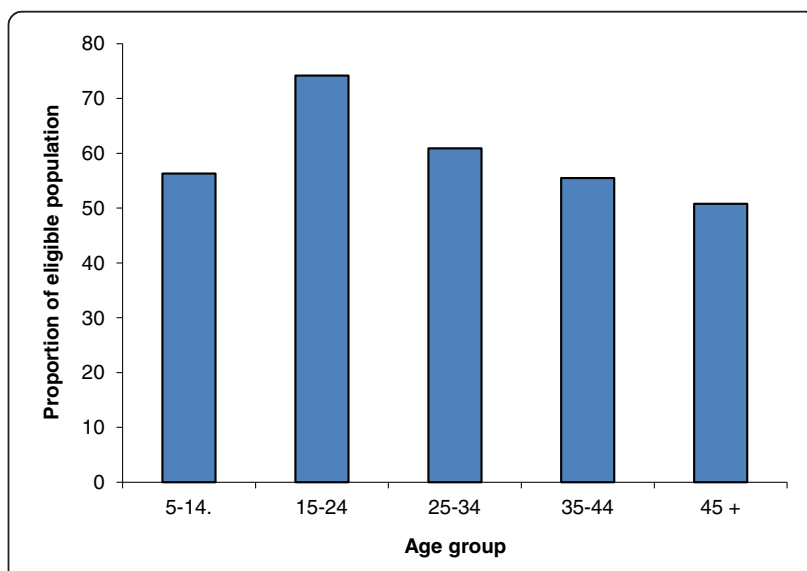

Figure 2 Proportion of eligible people (by age group) who did not take praziquantel as they were absent, Diéma, October 2008. 


\section{Treatment and side effects}

Side-effect data were available for 1,884 children and 1,640 mothers and teachers. During administration of the PZQ and ALB, side effects directly related to tablet administration (e.g., choking or coughing) were very rare (prevalence $<0.1 \%$ ) and not life threatening. In the ten villages, all side effects were investigated 24 hours after treatment. Most of the side effects were reported at varying prevalence levels, from $4.6 \%$ of children complaining about abdominal pain to $11.7 \%$ reporting fatigue. Headaches (5.1\%), vomiting (9.7\%) and diarrhoea (10.2\%) were also found at significant levels. By contrast, mothers reported very few children $(<1.0 \%)$ displaying any symptoms during the 24-hour follow-up.

\section{Discussion}

In the Diéma health district, the influence of social behaviour and cultural beliefs on public health interventions was greater than expected, especially among women. This is evident in the majority of the CDDs being men because women and, in particular younger ones, were not accepted for this position. In some villages, the two sexes could not mingle at a single meeting place.

One of the main findings was that many CDDs dropped out early due to a lack of incentive that was being provided (it was felt that the work load was too heavy for no financial compensation). Regarding the community interaction with the health services, the majority of the health workers did not visit villages regularly. Only $40 \%$ of the population reported that the District Director of Services (DDS) visited their communities. For $60 \%$ of the population, health workers visited the villages only during a specific campaign, such as immunisation and/or a cholera outbreak. Of those interviewed, $70 \%$ reported that the services provided in the communities are relevant to their needs and that they were satisfied with them. In terms of the community having an understanding of schistosomiasis and intestinal helminths, and their experiences with the treatment, it was found that $98 \%$ knew about the disease and $46 \%$ felt that it is a problem mainly because children who have the disease are constantly ill. The disease aetiology was attributed partly to a child's normal biological development (45\%), food (30\%) and contact with urine of those infected (20\%). Over half $(60 \%)$ of the population felt that schistosomiasis could be cured. Of those who believed it could be cured, $~ 50 \%$ thought that it could be treated with traditional medicine and the other half believed it could be done via modern medicine. With regards to prevention, $6 \%$ thought that the diseases caused by soil-transmitted helminths could be prevented, but no prevention was proposed for schistosomiasis.
Despite the health education about the transmission and prevention of the disease, standing water sources continued to be used by populations for fishing or for other domestic activities. After the rainy season, snails concentrate in small standing water sources with high levels of cercariae emerging. According to historical data, Diéma is known to be an endemic area for schistosomiasis haematobium [19] and our findings from the survey showed a high prevalence of $S$. haematobium $(78.4 \%)$ (data not presented here). In Niger, re-infection begun five months after treatment and the initial prevalence of infection decreased from $74.5 \%$ to $47.1 \%$ [20].

The mean coverage rate, especially in school children, achieved under community-directed intervention (CDI) was higher than that recommended by the World Health Organization [5]. Since 2002, the World Health Assembly, WHA 54.19, requires that by 2010, any round of anthelminthic treatment must be offered to at least $75 \%$ of children aged six to 15 years. Experience in community-directed treatment (ComDT) of onchocerciasis $[21,22]$ demonstrated that this treatment approach was not merely effective in the short term, but also proved to be sustainable in the long term. The success of the experience achieved in onchocerciasis led to extensions of ComDT to treatment of other disease control programmes, such as lymphatic filariasis [23] and schistosomiasis [8].

Though it is not yet known precisely what level of treatment coverage is required to achieve elimination of the disease, the coverage rate of $75 \%$, especially in school-age children, may be sufficient to interrupt transmission. Experience of parasite control programmes has shown that $75 \%$ coverage is an attainable target, delivering significant reductions in morbidity and preventing irreversible sequelae. The results of the census showed that coverage was higher in females than in males. This may be a result of the intense migration among males between the ages of 15-34 years travelling within neighbouring countries (Senegal, Cote d'Ivoire) or Europe and America. The income from these migrant males contributed to more than $80 \%$ of family budgets. With respect to the treatment prevalence, males and females were equal (76\%). Conversely, 15-29 year-old females in Cameroon were considered to be too busy with housekeeping activities and therefore less able to come to ivermectin distribution points [24]. However, in our study, the reasons for why eligible people did not receive PZQ were the same for males and females. The low coverage rate amongst 15-24-year olds (Figure 2) is often observed in this age group and tends to be related to economical concerns of this mobile population.

Absenteeism at the time of distribution was more common among Fulani and Moorish populations (70.3\%) who were primarily migrant cattle breeders 
compared to young Bambara/Sarakolle (30\%). The fact that some people (Fulani, Moorish and some resident Bambara/Sarakolle) were absent from villages at the time of distribution may be of epidemiological importance. These people, as well as migrants, remain to be important parasite reservoirs and may re-infect snails by shedding schistosome eggs upon return to the village. The Fulani and Moorish present a very different case. They live in separate settlements near the villages and they do not integrate within the Bambara and Sarakolle farm hamlets. They herd cattle in order to find pasture land, which explains why absenteeism at the time of distribution was a common reason for not receiving PZQ and ALB.

To meet CDI objectives, CDDs and health workers may need to pay more attention to ethnic diversity in order to build specific treatment schedules according to ethnic presence in the village. Even if the house-to-house mode distribution appeared to be efficient in this study, the success of this approach to increase coverage depends on the number of CDDs and their involvement in all aspects of distribution. Qualitative data helped to explain some findings. The following FGD comments reveal how villagers perceived drug distribution. Some said 'that the drug would affect them'. 'It makes [me] giddy. I didn't use [it] because of side effects'. Some individuals who were unable to be present for the drug distribution reported that they refused to take the drugs after hearing about their side effects. 'It causes stomach aches, vomiting and dizziness for those who use it. Some of them cannot work.' Overall, side effects occurred in $12.3 \%$ of the eligible participants (dizziness $-5.9 \%$, nausea, vomiting $-5.5 \%$ and others $-0.9 \%$ ). Some individuals initially rejected therapy but later took it 'because they got information from others that the drug was good'. 'It works.'

In-depth interviews with the Fulani or Moorish identified some of the reasons why their coverage rate was lower. 'We are migrants and most of us are following flocks and herds outside the village.' The native villagers, the Bambara and Sarakolle, confirmed the problem during the FDGs. 'At the time of the distribution, even if the Fulani and Moorish were present at hamlets (two to four kilometres from village), they did not move to the village for treatment but preferred to be treated in their own gomes'.

The role of community participation in improving coverage was identified by the CDDs during the FGDs. The comments by the CDDs distinctly differed to the present coverage status. The comments from those villages with low coverage were more negative: the community members were not involved in any other form of treatment besides the drug they took, they did nothing to assist during the distribution and the community did not cooperate as expected. 'I did not get any kind of assistance from anybody in carrying out the distribution.'

In high coverage villages, the following positive comments from the CDDs were common: 'the community members assisted in the area of health education, mobilisation and procurement of census materials; the community showed an interest in the programme as they helped me on the farm; community members brought a notebook for the census and a drug box; they fetched water when we were using the drug; they helped me to hold stick when I was distributing the drug'.

Besides these comments by the CDDs, village leaders, during the FGDs, typically gave the following comments, especially in low-coverage villages: 'In our village, most of the CDDs refused drug distribution because they prefer to be compensated for their participation, such as being noted in other programmes (i.e., HIV). Out of every five to six CDDs at baseline, only one or two of them finally accepted to do the job'. Sometimes, various programmatic communication problems were identified by the leaders and community members: 'On the distribution day, many residents were not around. Some men, and especially eligible children, took out their cattle for grazing'.

In high coverage villages, the role of the CDDs was judged to be positive: the CDDs showed an interest in the programme because their goal was to distribute drugs to all the villagers. When some community members were absent on the date of distribution, the CDDs returned twice or three times to the same family to meet them or waited for a period of time before returning to administer the drug. Even if people showed side effects, the CDDs encouraged them to use the drug, reassuring the villagers that the problems will eventually disappear. In fact, going from house to house was the best thing because they were able to reach even those who cannot walk. This was effective as people felt this was not interfering with their daily duties and because the CCDs were people they knew, they thus had faith in them.

\section{Conclusion}

The aim of the CDI approach was to provide better coverage rates, or at least to get them to be as good as the traditional programme-designed strategies (and also to ensure sustainability of long-term programmes). The findings of this study demonstrated that participatory methods could be incorporated in an effective control strategy for distribution of PZQ and ALB for treatment of schistosomiasis and intestinal helminthiasis. The most important factor for successful implementation of the CDI approach was primarily community mobilisation in order to get a high number of distributors devoted to the process. In all villages, community distributors 
would appreciate some form of incentive or compensation. More attention to the personal characteristics of the respondents and to community factors may also help raise the coverage rates.

\section{Additional file}

Additional file 1: Translation of the abstract into the six official working languages of the United Nations.

\section{Abbreviations}

ALB: Albendazole; APOC: African Programme for Onchocerciasis Control; CHC: Community health centre; CDD: Community drug distributors: CDHWs: Community-directed health workers; CDI: Community-directed intervention; CFSC: Communication for social change; Cl: Confident interval; ComDT: Community-directed treatment; DDS: District director of services; DHS: District health services; DMO: District medical officer; FGD: Focus group discussion; HIV: Human immunodeficiency virus; IRB: Institutional review board; OR: Odds ratio; PNLSHs: National Control Programme for Schistosomiasis and Soil-transmitted Helminths; PZQ: Praziquantel; WHA: World health assembly.

\section{Competing interests}

The authors declare that they have no competing interests.

\section{Authors' contributions}

DA participated in the conception and design of the study, data analysis and interpretation. He also contributed to the writing of the manuscript and assured the coordination of the trial. He has also reviewed the final version. BB participated in the design of the study and in onsite execution by collecting and analysing data. He also had all the clinical responsibility, contributed to the drug distribution and coordinated the field activities. KB participated in the conception and in onsite execution, assessment of side effects, data analysis and interpretation. He also supervised drug distribution, and contributed to the writing of the manuscript and in data analysing. SO participated in data collection, helped draft the manuscript, and analysed and reviewed the final version. DO participated in the conception and design of this paper. He contributed to the writing of the manuscript and the data analysis, participated in drafting of the paper and reviewed the final version. All authors read and approved the final manuscript.

\section{Acknowledgements}

We acknowledge the generous support provided by the UNDP/World/Bank/ WHO Special Programme for Research \& Training in Tropical Disease (TDR), the Regional Health Director and the District Directors of Health Services of Diéma and their respective Health Management Teams, the community leaders, the CDDs and the populations of the ten study villages for their role in the study, the research assistants for their input and dedication to the study, the staff of the Faculty of Medicine and Dentistry and all others, without whose support this study would not have been possible.

\section{Author details}

'Department of Epidemiology of Infectious Diseases, Faculty of Medicine, Pharmacy and Dentistry, UMI 3189, University of Sciences, Techniques and Technologies of Bamako, Box 1805, Bamako, Mali. ${ }^{2}$ High Institute for Training and Applied Research, Box 475E, Bamako, Mali.

\section{Received: 20 February 2013 Accepted: 13 May 2013}

Published: 10 June 2013

\section{References}

1. Steinmann P, Keiser J, Bos R, Tanner M, Utzinger J: Schistosomiasis and water resources development: systematic reviews, meta-analysis, and estimates of people at risk. Lancet Infect Dis 2006, 6:411-425.

2. Hotez P: Mass drug administration and integrated control for the world's high-prevalence neglected tropical diseases. Clin Pharmacol Ther 2009, 85:59-661.
3. Van der Werf MJ, De Vlas SJ, Brooker S, et al: Quantification of clinical morbidity associated with schistosome infection in sub-Saharan Africa. Acta Trop 2003, 86:125-139.

4. Brinkmann UK, Werler C, Traore M, Korte R: Experiences with mass chemotherapy in the control of schistosomiasis in Mali. Trop Med Parasitol 1988, 39:167-174.

5. Traoré M, Maude GH, Bradley DJ: Schistosomiasis haematobia in Mali: prevalence rate in school-age children as index of endemicity in the community. Trop Med Int Health 1998, 3(3):214-221.

6. Clements AC, Bosqué-Oliva E, Sacko M, Landouré A, Dembélé R, Traoré $M$, Coulibaly G, Gabrielli AF, Fenwick A, Brooker S: A comparative study of the spatial distribution of schistosomiasis in Mali in 1984-1989 and 20042006. PLoS Negl Trop Dis 2009, 3(5):e431.

7. World Health Organization Expert Committee: Prevention and control of schistosomiasis and soil-transmitted helminthiasis. World Health Organization Technical Reports Series 2002, 912:1-57.

8. CDI Study Group: Community-directed interventions for priority health problems in Africa: results of a multicounty study. Bull World Health Organ 2010, 88(7):509-518.

9. Mutalemwa P, Kisinza WN, Kisoka WJ, Kilima S, Njau J, Tenu F, Nkya T, Magesa SM: Community directed approach beyond ivermectin in Tanzania: a promising mechanism for the delivery of complex health interventions. Tanzanian Journal of Health Research 2009, 11:116-125.

10. Massa K, Magnussen P, Sheshe A, Ntakamulenga R, Ndawi B, Olsen A: The effect of the community-directed treatment approach versus the schoolbased treatment approach on the prevalence and intensity of schistosomiasis and soil-transmitted helminthiasis among schoolchildren in Tanzania. Trans R Soc Trop Med Hyg 2009, 103(1):31-37.

11. Seketeli A, Adeoye G, Eyamba A, Nnoruka E, Drameh P, Amazigo UV, Noma M, Agboton F, Aholou Y, Kale OO, et al: The achievements and challenges of the African Programme for Onchocerciasis Control (APOC). Ann Trop Med Parasitol 2002, 96(Suppl 1):S15-S28.

12. Sama MTH, Ngang P, Liese BH, Amazigo U, Seketeli A: A multi-centre study of community-directed ivermectin distributors' (CDDs') involvement in other healthcare and development programme activities in Cameroon, Togo, Sudan. Nigeria and Uganda. Tropical Doctor 2003, 33(4):237-241.

13. Anto F, Asoala V, Anyorigiya T, Oduro A, Adjuik M, Akweongo P, Aborigo R, Bimi L, Amankwa J, Hodgson A: Simultaneous administration of praziquantel, ivermectin and albendazole, in a community in rural northern Ghana endemic for schistosomiasis, onchocerciasis and lymphatic filariasis. Trop Med Int Health 2011, 16(9):1112-1119.

14. Njenga SM, Wamae CN, Njomo DW, Mwandawiro CS, Molyneux DH: Impact of tworounds of mass treatment with diethylcarbamazine plus albendazole in Malindi, Kenya. Trans R Soc Trop Med Hyg 2008, 102(10): 1017-1024.

15. Campagne G, Garba A, Bakire H, Vera C, Sidiki A, Chippaux JP: Continued ultrasonic follow-up of children infected with Schistosoma haematobium after treatment with praziquantel. Trop Med Int Health 2001, 6:24-30.

16. Werler C: Annual report of Schistosomiasis and Soil-transmitted Helminths National Control Programme (PNLSH). Bamako; 2007:38.

17. World Health organization: Preventive chemotherapy in human helminthiasis. A manual for health professionals and programme managers. Geneva: World Health Organization Report; 2006:74.

18. Katabarwa NM, Mutabazi D, Richards FOJR: Controlling onchocerciasis, ivermectin-treatment programmes in Uganda: why do some communities succeed and others fail? Ann Trop Med Parasitol 2000 94:343-352.

19. Dembélé R: Strategic plan for fighting against neglected tropical diseases (NTD) 2007-2011, Integration of mass chemotherapy strategy for trachoma, lymphatic filariasis, onchocerciasis, schistosomiasis and soil-transmitted helminths control. Report of Schistosomiasis and Soil-transmitted Helminths National Control Programme (PNLSH). Bamako; 2007-2011.

20. UNDP/World bankWHO Special Programme for Research and Training in Tropical Diseases (TDR): Community-Directed Treatment with Ivermectin. Geneva: World Health Organization; 1996.

21. African Programme for Onchocerciasis Control: Community-Directed Treatment with Ivermectin (CDTI). Ouagadougou: A Practical Guide for trainers of Community-Directed Distributors. APOC; 1998. 
22. UNDP/World Bank/WHO Special Programme for Research and Training in Tropical Diseases (TDR: Community-Directed Treatment of Lymphatic Filariasis in Africa. Geneva; 2000. TDR/IDE/RP/CDTI/00.2.

23. World Health Organization: Report of a multi-centre study in Ghana and Kenya. Geneva: World Health Organization; 2000.

24. Boussinesq M, Chippaux JP, Ernould JC, Quillevere D, Prod'hom J: Effect of repeated treatments with ivermectin on the incidence of onchocerciasis in northern Cameroon. AmJTrop Med Hyg 1995, 53:63-67.

doi:10.1186/2049-9957-2-11

Cite this article as: Dabo et al:: Factors associated with coverage of praziquantel for schistosomiasis control in the community-direct intervention (CDI) approach in Mali (West Africa). Infectious Diseases of poverty 2013 2:11.

\section{Submit your next manuscript to BioMed Central and take full advantage of:}

- Convenient online submission

- Thorough peer review

- No space constraints or color figure charges

- Immediate publication on acceptance

- Inclusion in PubMed, CAS, Scopus and Google Scholar

- Research which is freely available for redistribution 